\title{
miR-370 regulates cell proliferation and migration by targeting EGFR in gastric cancer
}

\author{
TAO NING ${ }^{*}$, HAIYANG ZHANG ${ }^{*}$, XINYI WANG ${ }^{*}$, SHUANG LI, LE ZHANG, TING DENG, LIKUN ZHOU, \\ RUI LIU, XIA WANG, MING BAI, SHAOHUA GE, HONGLI LI, DINGZHI HUANG, \\ GUOGUANG YING and YI BA
}

Tianjin Medical University Cancer Institute and Hospital, National Clinical Research Center for Cancer, Key Laboratory of Cancer Prevention and Therapy, Tianjin, Tianjin's Cinical

Research Center for Cancer, Tianjin 300060, P.R. China

Received October 10, 2016; Accepted December 30, 2016

DOI: 10.3892/or.2017.5660

\begin{abstract}
Epidermal growth factor receptor (EGFR) is known to be overexpressed in $\sim 30 \%$ of gastric cancer (GC) cases, and may serve as an effective biomarker for predicting the clinical benefit of anti-EGFR therapy. However, the mechanism underlying the regulation of EGFR expression remains unknown. Evidence indicates that post-transcriptional regulation may exist in the process of EGFR expression. In the present study, we aimed to explore whether miR-370 is involved in this process, and how it impacts the biological behaviors of GC cells. In the present study, we first determined the role of EGFR in GC by means of an EGFR overexpression plasmid and siRNAs. Then, the expression levels of EGFR protein and mRNA in GC tissues were analyzed through immunohistochemistry and quantitative RT-PCR (RT-qPCR). Bioinformatics tools and dual-luciferase assay were applied to predict and validate the relevant miRNA targeting EGFR. Finally, human GC MGC-803 cells were selected to explore the effect of miRNA on cell proliferation and migration. We found that suppression of EGFR inhibited the proliferation and migration of GC cells. In addition, the levels of EGFR protein in the GC tissues were $\sim 4$ times higher than that in the corresponding paired non-cancerous tissues while the mRNA levels of EGFR were only $\sim 2$-fold as high as that in the adjacent non-cancerous tissues. Bioinformatics tools predicted that miRNA-370 was a regulator of EGFR and dual-luciferase
\end{abstract}

Correspondence to: Dr Yi Ba, Tianjin Medical University Cancer Institute and Hospital, National Clinical Research Center for Cancer, Key Laboratory of Cancer Prevention and Therapy, Tianjin, Tianjin's Cinical Research Center for Cancer, 18 Huan Hu Xi Road, Tianjin 300060, P.R. China

E-mail: bayi@tjmuch.com

${ }^{*}$ Contributed equally

Key words: miR-370, gastric cancer, EGFR, cell proliferation, migration assay validated that miR-370 could directly bind to the 3'-untranslated region (3'-UTR) of EGFR mRNA. Meanwhile, an inverse correlation between miR-370 and EGFR was found in the GC tissues. Overexpression of miR-370 suppressed the proliferation and migration of GC cells, while downregulation of miR-370 promoted proliferation and migration. The present study may provide new information for understanding the molecular mechanism underlying the regulation of EGFR protein expression in GC and may be of important clinical significance to guide targeted therapy.

\section{Introduction}

Gastric cancer (GC) is one of the most common malignancies, ranking second, and is the fourth leading cause of cancer-related mortality in China (1). Although the survival time of patients with GC has improved during the past decade, prognosis remains poor for patients with advanced GC. The 5 -year survival rate for patients with localized disease is $\sim 60 \%$, and only $3-5 \%$ for those with distant metastasis $(2,3)$. In consideration of the low efficacy of traditional chemotherapy and radiotherapy, molecular-targeted therapy, due to its high efficiency, is an attractive therapeutic strategy (4).

The epidermal growth factor receptor $(E G F R)$ gene is located at the chromosomal region $7 \mathrm{p} 12$ and encodes a $70-\mathrm{kDa}$ transmembrane tyrosine kinase receptor that contributes to cancer progression by mediating cellular proliferation, migration, adhesion and metastasis (5). Approximately 30\% of GC patients are reported to show EGFR protein overexpression, and thus EGFR signaling pathways serve as attractive therapeutic targets $(6,7)$. However, to date, all the agents targeting EGFR have failed to demonstrate any antitumor efficacy for treating GC patients in the clinic, presumably since no precise patient inclusion criteria were set for the clinical trials (8). Intriguing retrospective biomarker analyses of the clinical trials suggest that a subpopulation of tumors with EGFR gene copy number gain or protein overexpression may benefit from anti-EGFR therapy, implying that refining the EGFR biomarker, may yet yield positive results (9). EGFR protein expression or gene amplification may serve as an effective biomarker for predicting the clinical benefit of anti-EGFR 
therapy $(9,10)$. However, various studies have shown that the levels of EGFR gene amplification are not always consistent with those of the protein expression in $\mathrm{GC}$ which indicate that a post-transcriptional regulation may exist $(11,12)$. Further in detail research of the regulatory mechanism underlying the EGFR protein expression in GC may be of important clinical significance to guide targeted therapy.

MicroRNAs (miRNAs) are a class of endogenous, noncoding, single-stranded RNAs ( $22 \mathrm{bp})$ that regulate gene expression by directly binding with the 3'-untranslated region (3'-UTR) of target mRNAs, causing degradation of the specific mRNA sequence or translational inhibition at the post-transcriptional level (13). Research indicates that over $30 \%$ of human genes are regulated by miRNAs $(14,15)$. Acting as oncogenes or tumor suppressors, miRNAs can repress the expression of cancer-related genes (16). Several studies have reported that alterations in miRNA levels occur in various human tumors, including GC, where miRNAs are related to several aspects of cancer pathogenesis including self-renewal, invasion and metastasis by targeting EGR2 (17), MAPK (18) and PI3K/AKT signaling pathways (19). Therefore, we speculate that specific miRNAs may participate in the regulation of EGFR protein expression in GC.

In a previous study, miR-370 was reported to function as a tumor suppressor in colorectal cancer by suppressing EGFR signaling (20). It has been reported that miR-370 is also significantly downregulated in GC (21). However, whether EGFR protein expression is regulated by miR-370 in GC remains unknown. In the present study, we used immunohistochemistry (IHC), western blotting and quantitative RT-PCR (RT-qPCR) assay to study the relationship between the EGFR protein and the mRNA level in GC tissues. We also used bioinformatics tools and dual-luciferase assay to identify and validate the potential miRNA for targeting EGFR. Finally, a series of functional experiments in vitro were carried out to confirm the role of miR-370. We aimed to investigate whether EGFR protein overexpression in GC is modulated by miR-370 at the post-transcriptional level and how it may impact the biological behavior of GC cells.

\section{Materials and methods}

Human tissues. Human GC and paired adjacent non-cancerous tissues were derived from patients undergoing radical surgery at the Tianjin Medical University Cancer Institute and Hospital (Tianjin, China). Both tumor and non-cancerous tissues were confirmed histologically and the pathological type of cancer was adenocarcinoma. Written informed consent was obtained in all cases, and all aspects of the present study were approved by the Ethics Committee of Tianjin Medical University Cancer Institute and Hospital.

Cell line and culture. The human GC cell line MGC-803 was purchased from the Shanghai Institute of Cell Biology, Chinese Academy of Sciences (Shanghai, China). The human embryonic kidney epithelial cell line HEK293T was obtained from the American Type Culture Collection (ATCC; Manassas, VA, USA). MGC-803 and HEK293T cells were cultured in Dulbecco's modified Eagle's medium (DMEM) supplemented with 10\% fetal bovine serum (FBS) (both from
Gibco, Carlsbad, CA, USA), with routine addition of ampicillin and streptomycin.

Cell transfection. MGC-803 cells were seeded in 6-, 12- or 24-well plates, and transfected with Lipofectamine 2000 (Invitrogen, Carlsbad, CA, USA) $24 \mathrm{~h}$ later according to the manufacturer's instructions. Equal amounts of miR-370 mimics, inhibitors and negative control (RiboBio, Guangzhou, China) were used for miRNA overexpression and downregulation. miR-370 mimics are small, chemically modified double-stranded RNAs that mimic endogenous miR-370 and enable miR-370 functional analysis by upregulation of miR-370 activity. miR-370 inhibitors are small, chemically modified single-stranded RNA molecules designed to specifically bind to and inhibit endogenous miR-370 molecules and enable miR-370 functional analysis by downregulation of miR-370 activity. For the purpose of EGFR upregulation, an EGFR overexpression plasmid without miR-370-responsive 3'-UTR was put into use and an empty plasmid was used as a negative control. Two siRNAs targeting human EGFR were used for EGFR downregulation (EGFR siRNA-1, AACACAGUGGA GCGAAUUCCU; EGFR siRNA-2, CGCAAAGUGUGUAAC GGAAUA) and also a scrambled siRNA (both from RiboBio) was used as a negative control.

miRNA target prediction and dual-lufierase reporter assay. miRNAs potentially targeting the 3'-UTR of EGFR were identified using 3 internet-based bioinformatic algorithms from miRanda (http://www.microrna.org/) (22), TargetScan (http:// www.targetscan.org/) (23) and PicTar (http://pictar.mdc-berlin. $\mathrm{de} /$ ) (24). Target recognition is based on complementarity of the nucleotide sequence to the 3'-UTR region of EGFR mRNA. The miRNAs that were predicted by at least 2 of the algorithms to bind were accepted as candidates for further study. miRNA expression data were obtained from microRNA.org (http:// www.microrna.org/). The predicted 3'-UTR miR-370 targeting region was inserted into the pMIR-REPORT plasmid (Ambion, Austin, TX, USA) and was then affirmed by DNA sequencing. Reporter assays were performed $36 \mathrm{~h}$ post-transfection using the Dual-Luciferase Assay System (Promega, Madison, WI, USA). The intensity of firefly luciferase was normalized to Renilla luciferase.

Protein extraction and western blotting. Proteins were extracted from the human tissues and cultured cells in lysis buffer added with a protease inhibitor. Total cell lysates were separated on SDS-PAGE gels and transferred to polyvinylidene fluoride (PVDF) membranes (Roche, Mannheim, Germany). After blocking with $2 \%$ bovine serum albumin (BSA), the membrane was incubated with anti-EGFR (mouse, monocolonal) and anti-GAPDH (mouse, monocolonal) (both from Santa Cruz Biotechnology, Inc., Santa Cruz, CA, USA) antibodies overnight at $4^{\circ} \mathrm{C}$. Then the membranes were incubated with the secondary antibodies and visualized with an enhanced chemiluminescencent horseradish peroxidase (HRP) substrate kit (Millipore, Billerica, MA, USA) according to the manufacturer's instructions.

$R N A$ isolation and RT-qPCR. Total RNA was isolated from the cultured cells and tissues using TRIzol reagent (Invitrogen) 
A

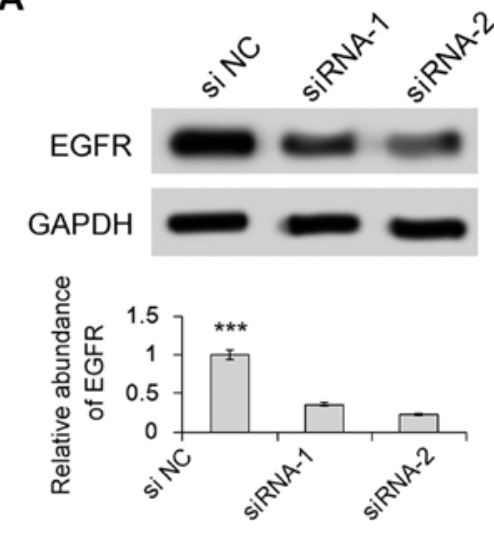

C

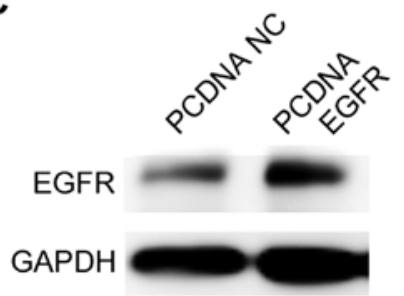

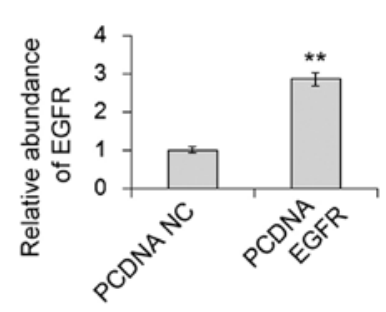

B

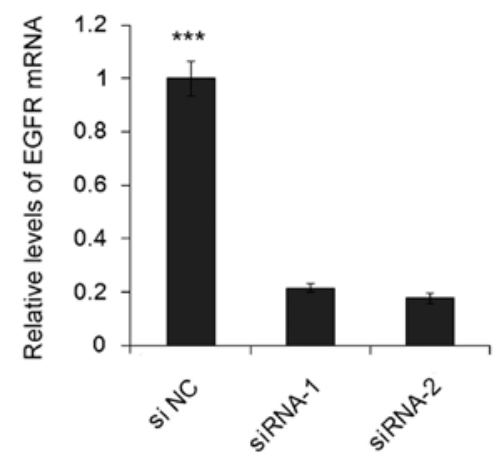

D

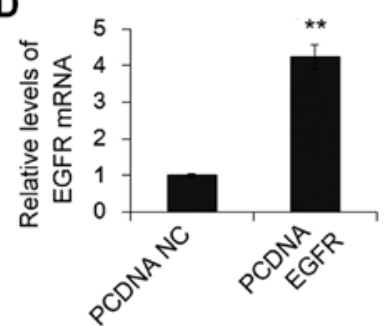

Figure 1. Effects of siRNAs or overexpression plasmid on the EGFR protein expression in GC cells. (A) Western blot assay of the EGFR protein expression in MCG-803 cells transfected with siRNAs $(n=3)$. (B) Quantitative RT-PCR analysis of the relative levels of EGFR mRNA in MGC-803 cells transfected with siRNAs ( $n=3)$. (C) Western blot assay of the EGFR protein expression in MCG-803 cells transfected with the overexpression plasmid ( $\mathrm{n}=3$ ). (D) Quantitative RT-PCR analysis of the relative levels of EGFR mRNA in MGC-803 cells transfected with the overexpression plasmid ( $\mathrm{n}=3$ ). si NC refers to control scrambled siRNA, siRNA-1 refers to EGFR siRNA-1, siRNA-2 refers to EGFR siRNA-2, PCDNA NC refers to control empty plasmid, PCDNA EGFR refers to the overexpression plasmid; ${ }^{* *} \mathrm{p}<0.01 ;{ }^{* * *} \mathrm{p}<0.001$.

according to the manufacturer's protocol. The RNA concentration was quantified using a NanoDrop 1000 spectrophotometer (Thermo Fisher Scientific, Waltham, MA, USA). The cDNA was synthesized from $1 \mu \mathrm{g}$ of total RNA using primers specific to EGFR and GAPDH in conditions as follows: $16^{\circ} \mathrm{C}$ for $15 \mathrm{~min}, 42^{\circ} \mathrm{C}$ for $60 \mathrm{~min}$ and $85^{\circ} \mathrm{C}$ for $5 \mathrm{~min}$. The quantity of miR-370 was analyzed using TaqMan miRNA probes (Applied Biosystems, Foster City, CA, USA). The PCR was initiated by a 5 -min hold at $95^{\circ} \mathrm{C}$, followed by 40 cycles of denaturation at $95^{\circ} \mathrm{C}$ for $15 \mathrm{sec}$ and annealing/extension at $60^{\circ} \mathrm{C}$ for $1 \mathrm{~min}$. All the reactions were run in triplicate. U6 snRNA was used as an internal control for miRNA. The mRNA level of EGFR was normalized to GAPDH. The $\Delta \mathrm{Ct}$ (cycle threshold) method was applied to calculate the relative level of the target gene that was normalized to the control through the equation: $2^{-\Delta \mathrm{Ct}}$, in which $\Delta \mathrm{Ct}=\mathrm{Ct}_{\text {gene }}-\mathrm{Ct}_{\text {control }}$. Primer sequences for EGFR and GAPDH were as follows: EGFR sense, 5'-TTGCC GCAAAGTGTGTAACG-3' and EGFR antisense, 5'-GTCAC CCCTAAATGCCACCG-3'; GAPDH sense, 5'-AGAAGGCT GGGGCTCATTTG-3' and GAPDH antisense, 5'-AGGGGC CATCCACAGTCTTC-3'.

Cell proliferation assay. MGC-803 cells were seeded in 24-well plates $\left(4 \times 10^{4}\right.$ cells/well) and transfected with miR-370 mimics, inhibitors, EGFR overexpression plasmid, EGFR siRNAs or the relevant negative controls. Twenty-four hours after transfection, a Cell-Light 5-ethynyl-2'-deoxyuridine (EdU) Apollo DNA cell kit (RiboBio) was used to measure the cell proliferation ability according to the manufacturer's instructions as previously described (25). Briefly, EdU at a concentration of $50 \mu \mathrm{M} / \mathrm{ml}$ was added into the medium and cultured for $5 \mathrm{~h}$. Then, the cells were fixed with $4 \%$ paraformaldehyde for $30 \mathrm{~min}$ and treated with $0.5 \%$ Triton X-100 for $15 \mathrm{~min}$. After being washed in phosphate-buffered saline (PBS), the cells were incubated in darkness with a fluorescent dye of Apollo which was incorporated with EdU for $30 \mathrm{~min}$, and then repeatedly washed. The cell nuclei were then stained by Hoechst 33342 for another $30 \mathrm{~min}$. Eventually, EdU-labeled cells were counted manually in 5 fields of view randomly selected from each well. The EdU-labeling index was calculated as the ratio of EdU add-in cells to Hoechst 33342-stained cells. All of the stainings were performed in triplicate.

Transwell cell migration assay. The capability of cell migration was assessed using a 24-well Boyden chamber with an 8.0- $\mu \mathrm{m}$ pore polycarbonate membrane insert (Corning, Corning, NY, USA) according to the manufacturer's instructions. At $24 \mathrm{~h}$ post-transfection, $\sim 10^{5}$ cells were seeded in the upper chamber with $200 \mu \mathrm{l}$ serum-free medium. Simultaneously, $600 \mu \mathrm{l}$ medium with $10 \%$ FBS was added into the lower chamber for purpose of chemotaxis. Another $24 \mathrm{~h}$ later, the membranes were fixed with methanol and stained with a 3-Step Stain Set (Thermo Scientific, UK). All assays were performed in triplicate. To minimize the bias, at least 3 randomly selected fields were counted at a magnification of $\mathrm{x} 200$, under a microscope.

Wound healing assay. MGC-803 cells were seeded into 12-well plates, and transfected using the method as described in 'Cell transfection'. The day after transfection, when the density of the cells was over $90 \%$, each well was scratched 
A

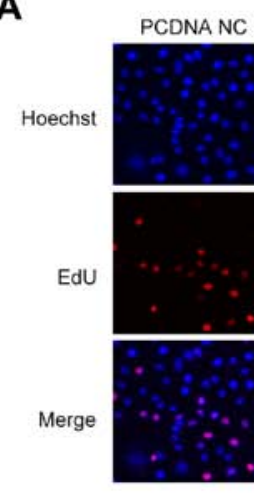

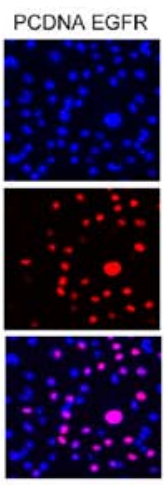

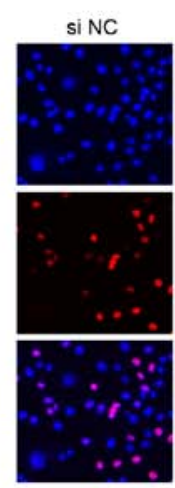

C

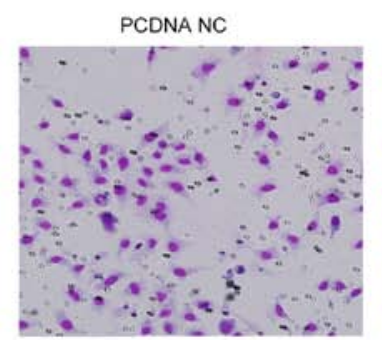

si NC
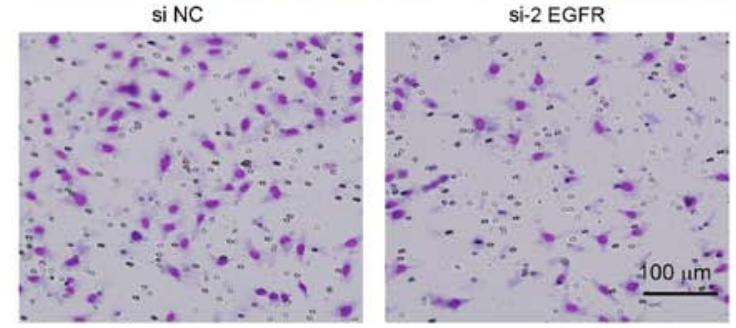

PCDNA EGFR

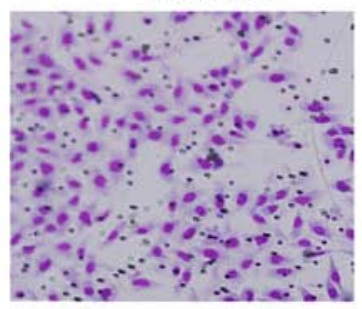

si-2 EGFR
B
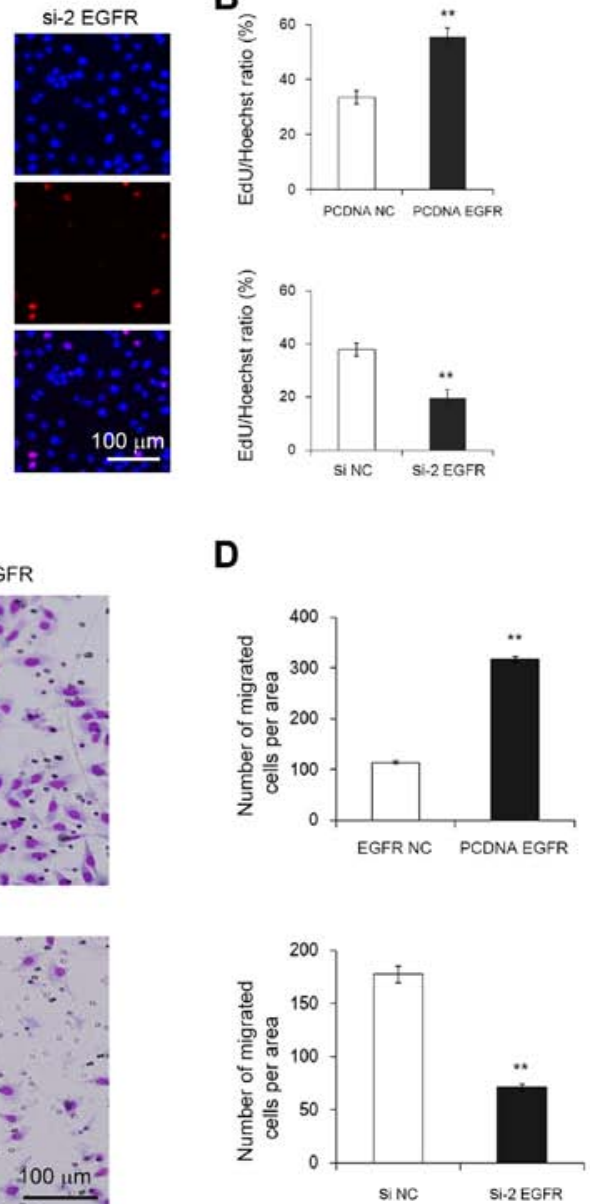

E
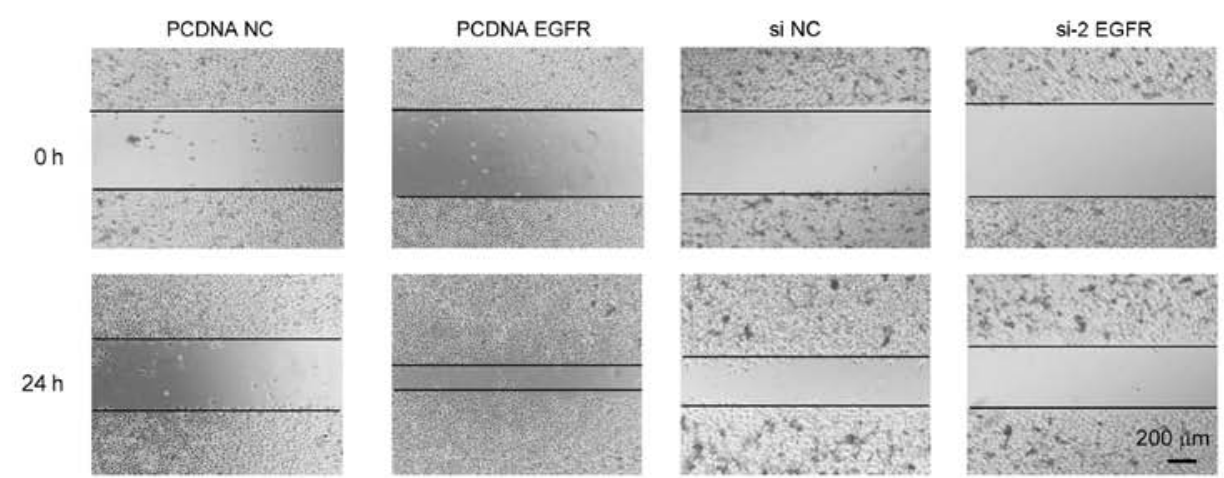

Figure 2. Effect of EGFR overexpression or silencing on the cell proliferation and migration in GC cells. (A) EdU assay demonstrated that overexpression of EGFR promoted cell proliferation, whereas knockdown of EGFR inhibited cell proliferation. (B) Quantitative analysis of A ( $n=3$ ). (C) Transwell assay showed that overexpression of EGFR enhanced cell migration, whereas silencing of EGFR inhibited cell migration. (D) Quantitative analysis of $C(n=3)$. (E) Evaluation of EGFR-mediated cell migration using wound healing assay. PCDNA NC refers to the control empty plasmid, PCDNA EGFR refers to the overexpression plasmid, si NC refers to control scrambled siRNA, and Si-2 EGFR refers to EGFR siRNA-2; ${ }^{* *}$ p $<0.01$.

to create 2 empty linear regions with a $10-\mu 1$ pipette tip. Subsequently, the cells were cultured in DMEM without FBS in a humidified incubator. The wound healing was observed and imaged at 0,12 and $24 \mathrm{~h}$ after scraping. The distance of the wound zone was measured for at least 3 randomly selected fields.

IHC assay.Formalin-fixed paraffin-embedded specimens of both $\mathrm{GC}$ and paired adjacent non-cancerous tissues were sectioned $(8-\mu \mathrm{m}$ thick) and stained with an anti-EGFR antibody (mouse, monocolonal; Santa Cruz Biotechnology) at a 1:100 dilution. The 3,3'-diaminobenzidine (DAB) system (Zhongshanjinqiao, Beijing, China) was applied to confirm the positive staining. Six random fields were selected for each specimen.

Statistical analysis. SPSS Statistics 20.0 (SPSS, Inc., Chicago, IL, USA) was used to analyze the data. Differences between groups were tested by analysis of variance and an unpaired Student's t-test. $\mathrm{p}<0.05$ was considered as statistical significance. 
A
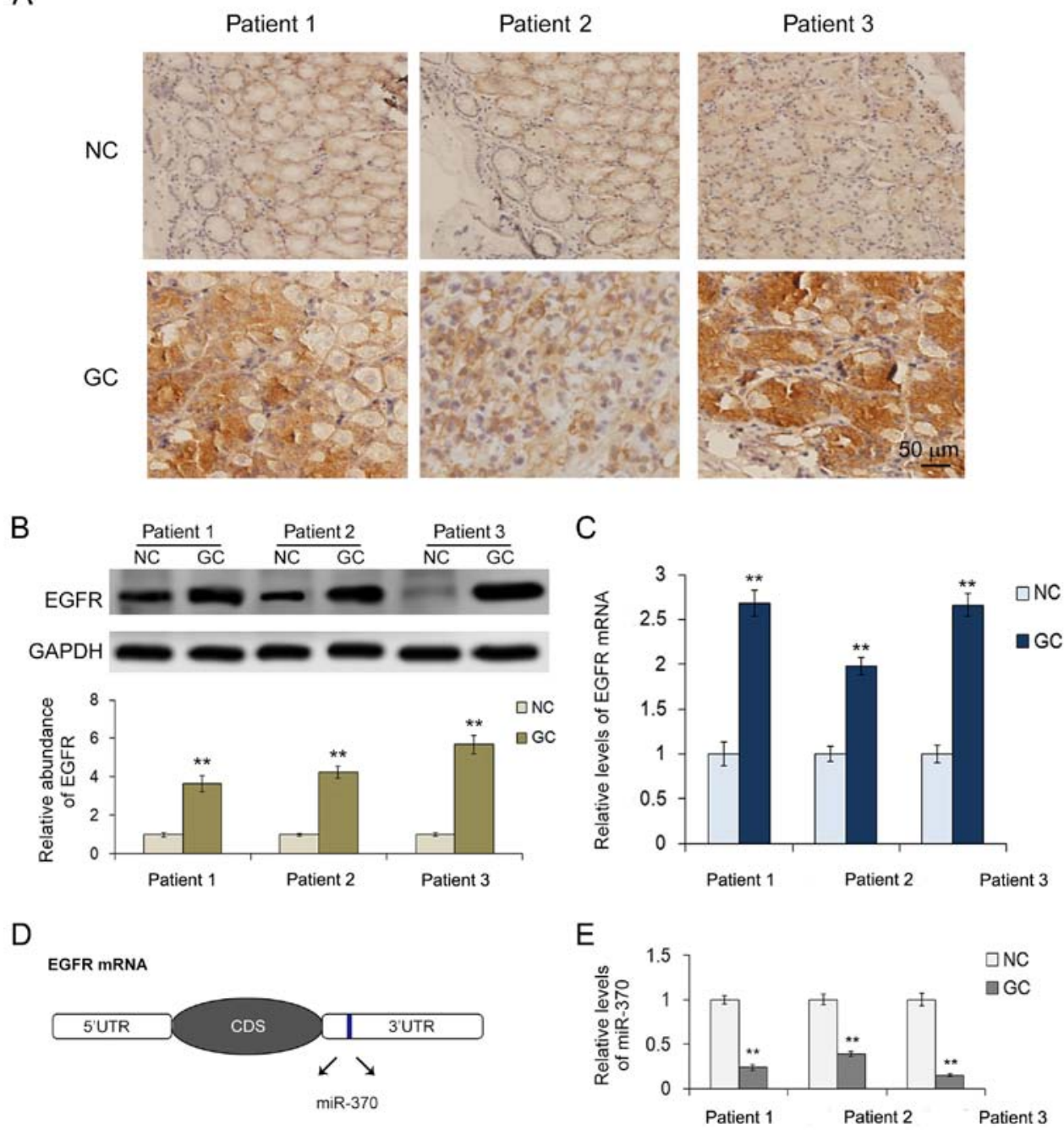

Figure 3. Expression patterns of EGFR and miR-370 in GC tissues. (A) Immunohistochemical staining of human GC and paired non-cancerous tissues (magnification, x400). (B) Western blot analysis of EGFR protein expression in the GC cancer tissues and paired non-cancerous tissues. (C) Relative EGFR mRNA levels in the GC tissues. (D) The predicted binding region of miR-370 in the mRNA of EGFR using publicly available computational algorithms. (E) Relative levels of miR-370 in the GC and paired non-cancerous tissues. GC indicates gastric cancer tissues, NC indicates paired non-cancerous tissues. The experiments were repeated at least 3 times, and error bars represent mean $\pm \mathrm{SD} ;{ }^{* *} \mathrm{p}<0.01$.

\section{Results}

Biological role of EGFR in GC cells. We first investigated the effect of EGFR on cell proliferation and migration in GC cells. Plasmid and 2 siRNAs were used to overexpress or knock down EGFR in the MGC-803 cells. As shown in Fig. 1A-D, the expression levels of both EGFR protein and mRNA were markedly upregulated by the plasmid and were downregulated by the siRNAs as compared with the normal control. MGC-803 cells in which EGFR protein was overexpression exhibited a higher rate of proliferation and increased migration, whereas the cells with knockdown of EGFR exhibited a notably lower rate of proliferation and decreased migration (Fig. 2A-E). Thus, EGFR acts as a cancer promoter in GC and its downregulation decreased the proliferation and migration of cancer cells.

Identification of miR-370 as a potential regulator of EGFR. To further understand the expression regulation of EGFR, IHC was applied to analyze the expression pattern of EGFR protein in GC. Ten pairs of GC and adjacent non-cancerous tissues derived from patients undergoing radical gastrectomy were collected in the present study. Results showed that 3 patients had high EGFR protein expression in the GC tissues compared with the paired non-cancerous tissues (Fig. 3A). These 3 patient tissues were further analyzed using western blotting and RT-qPCR. As shown in Fig. 3B, the levels of EGFR protein in the GC tissues were $\sim 4$ times higher than that in the corresponding paired non-cancerous tissues. However, the mRNA levels of EGFR were only 2 -fold as high as that of the normal control (Fig. 3C). The disparity between the protein and the mRNA suggests that expression of EGFR in GC may be regulated at a post-transcription level. As miRNA-mediated regression of mRNA transcripts is thought to be one of the most important modes of post-transcriptional regulation, 3 publicly available computational algorithms were then used to predict the potential miRNAs targeting EGFR. Both miRanda and TargetScan predictions showed that the 3'-UTR of EGFR mRNA contains a potential miR-370-binding site (Fig. 3D). To identify the relationship between miR-370 and EGFR in the 
A

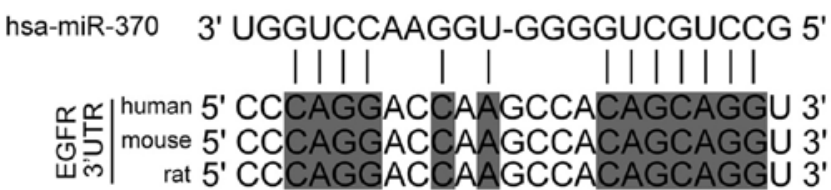

B

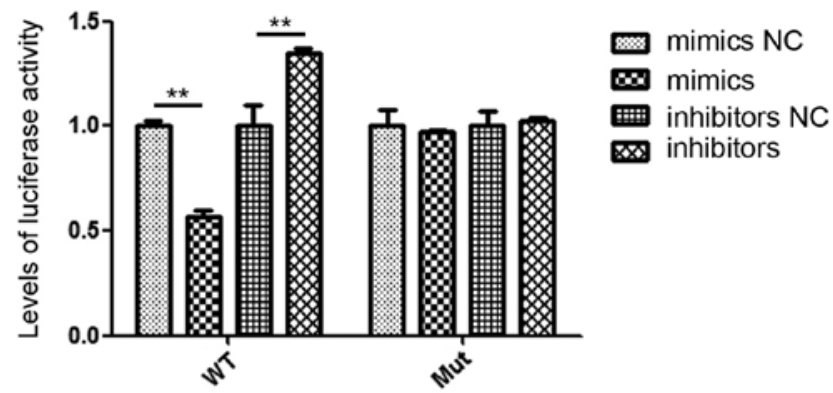

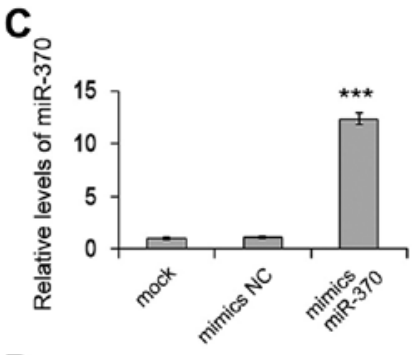

D

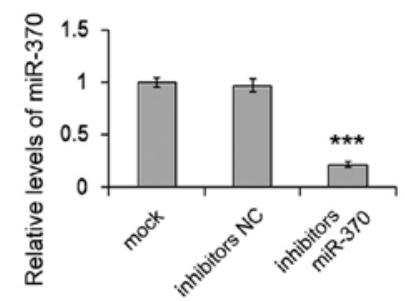

E
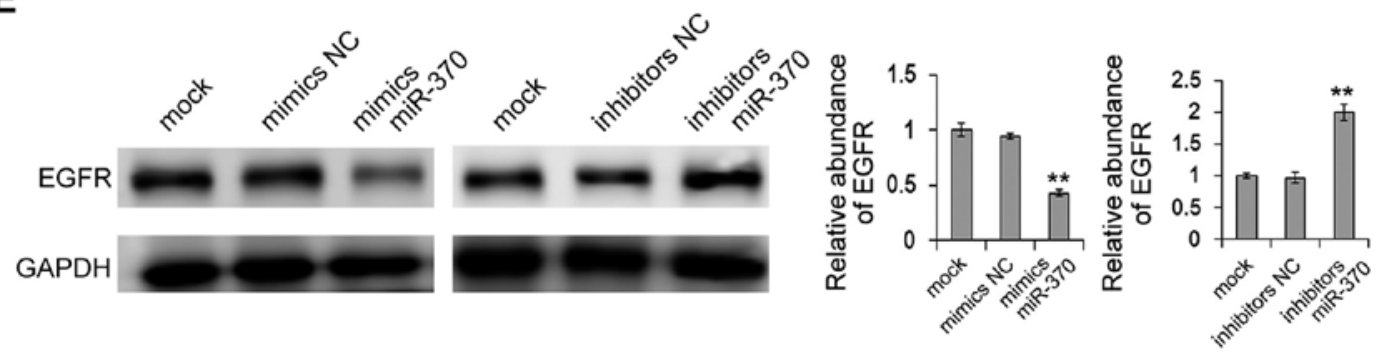

F
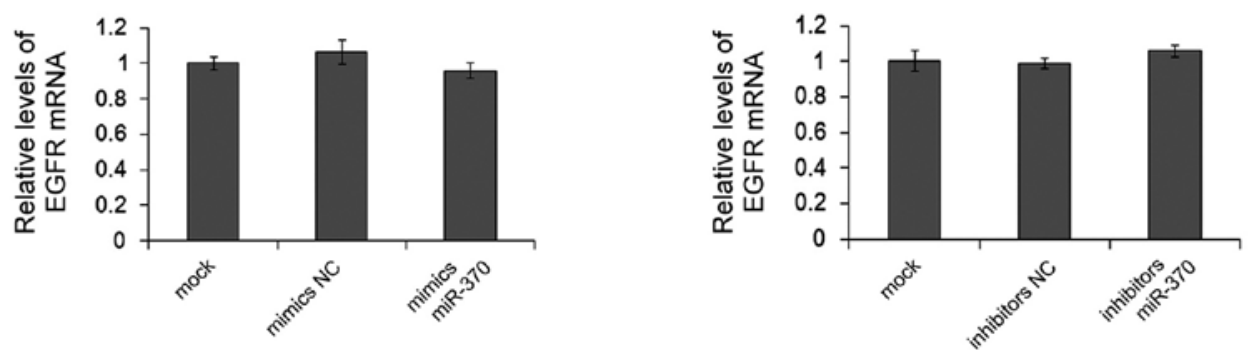

Figure 4. Effect of miR-370 on the expression of EGFR in GC cells. (A) Schematic description of the base-pairing interaction between miR-370 and EGFR mRNA. (B) Direct recognition of the EGFR 3'-UTR by miR-370. HEK293T cells were cotransfected with dual-luciferase reporters containing either wild-type or mutant EGFR 3'-UTR with miR-370 mimics and inhibitors (n=3). (C and D) Quantitative RT-PCR analysis of the relative miR-370 levels in MGC-803 cells transfected with mimics or inhibitors $(n=3)$. (E) Western blot assay of the suppression of EGFR protein expression by miR-370 in MGC-803 cells ( $\mathrm{n}=3$ ). (F) Quantitative RT-PCR analysis of EGFR mRNA expression in MGC-803 cells ( $\mathrm{n}=3)$. Mock refers to parental cells, mimics NC refers to control mimics, inhibitors NC refers to control inhibitors; ${ }^{* *} \mathrm{p}<0.01,{ }^{* * *} \mathrm{p}<0.001$.

GC, we further explored the levels of miR-370 in the 3 pairs of GC and the corresponding paired non-cancerous tissues. As expected, miR-370 showed an obvious decrease in all of the tumor tissues (Fig. 3E). Thus, we proposed that miR-370 is a regulator of EGFR in GC and we selected miR-370 for further experiments.

Validation of miR-370 as a regulator of EGFR. The levels of miR-370 and EGFR protein showed an inverse correlation in the GC tissues, and the prediction by bioinformatics suggested that miR-370 is a potential regulator of EGFR. We then further validated miR-370 as a regulator of EGFR. As shown in Fig. 4A, bioinformatics results showed that miR-370 can bind with 3'-UTR of EGFR mRNA with 7-mer seeds and this putative miR-370-binding site and its adjacent sequences are highly conserved among vertebrates. Dual-luciferase assay showed that the relative levels of luciferase activity of the reporter gene were significantly inhibited when HEK293T cells were cotransfected with miR-370 mimics and the luciferase reporters containing the predicted target regions of EGFR mRNA, while the inhibition was lost when the binding sites in 3'-URT were mutated (Fig. 4B). Next, relative levels of miR-370 and the expression of EGFR protein and mRNA in the MGC-803 cells were also respectively detected, using RT-qPCR or western blot analysis following transfection of mimics or inhibitors. As shown in Fig. 4C and D, miR-370 mimics significantly increased the miR-370 levels in the MGC-803 cells, while miR-370 inhibitors markedly downregulated the miR-370 levels. Overexpression of miR-370 led to a reduction in EGFR protein, whereas inhibition of miR-370 led to enhanced expression of the EGFR 
A

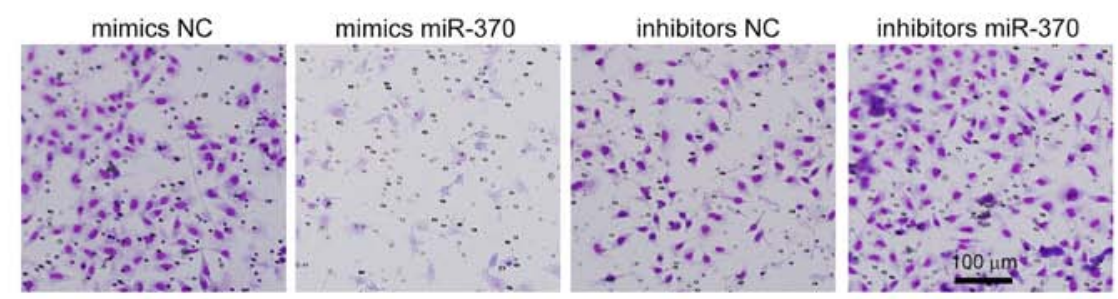

B

C
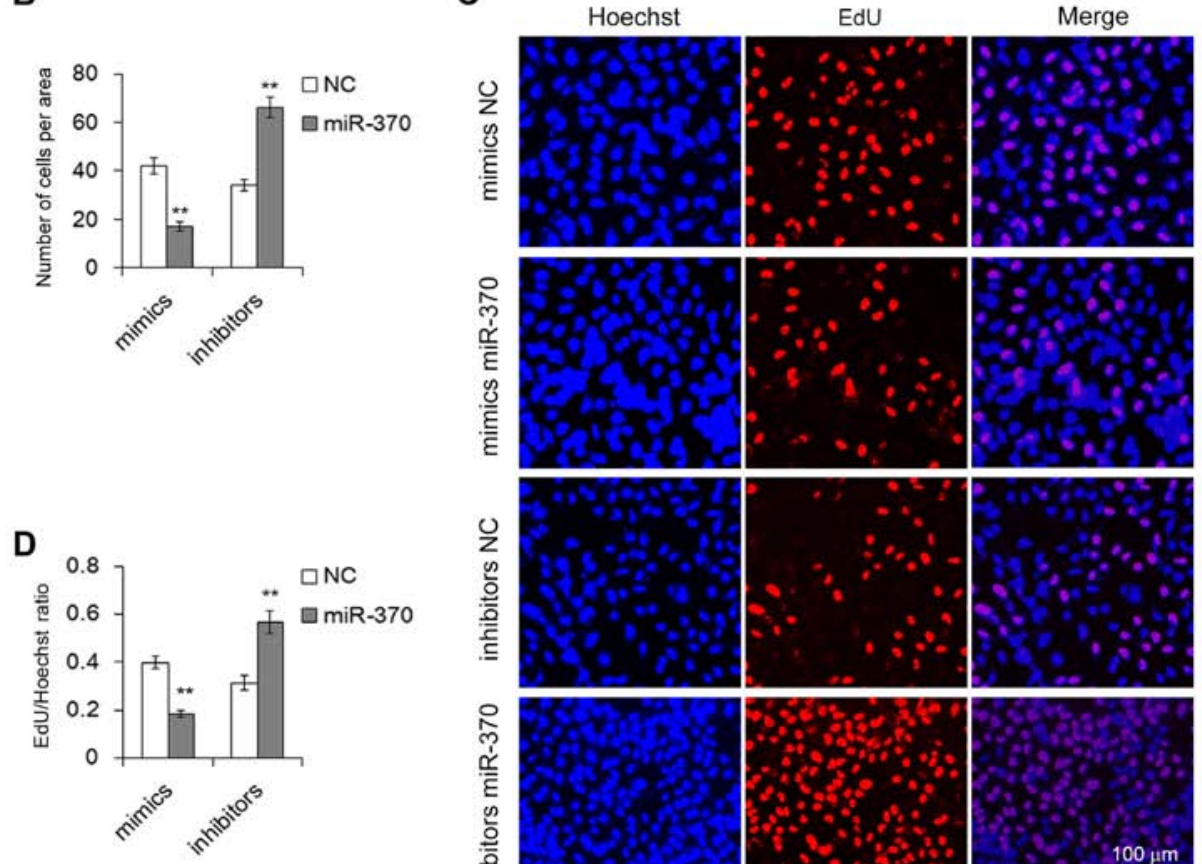

E
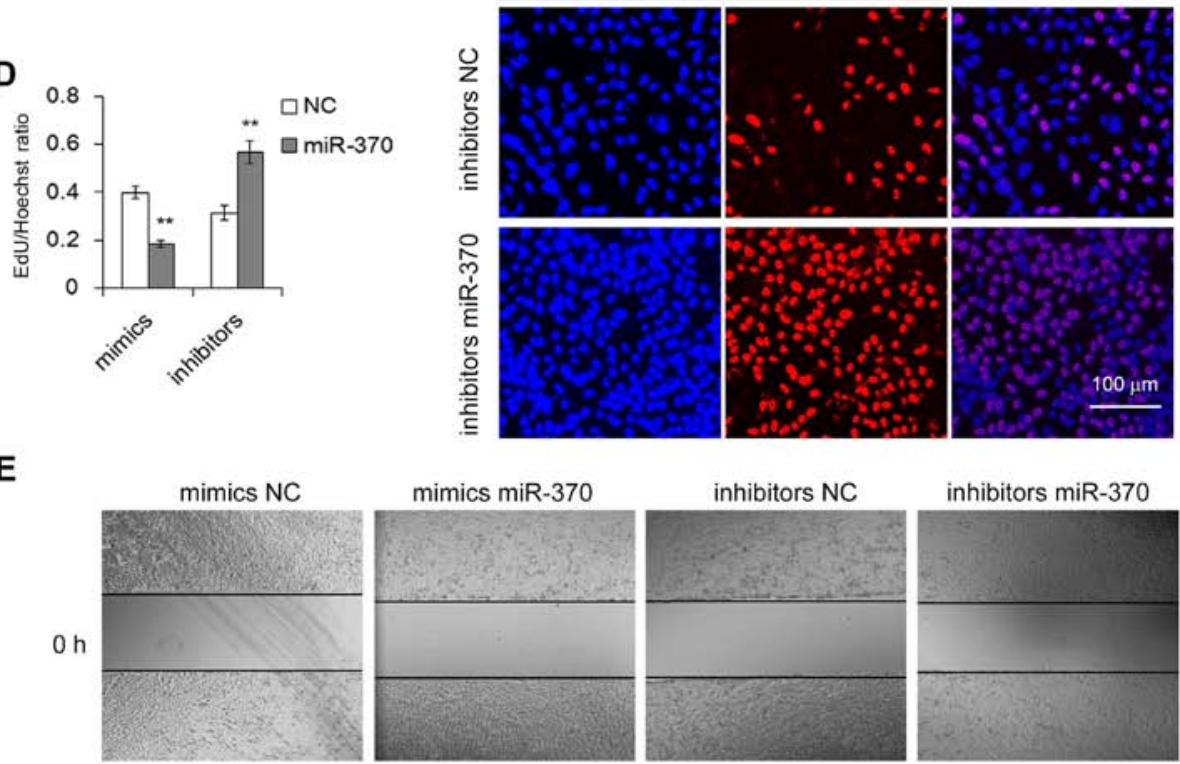

inhibitors miR-370
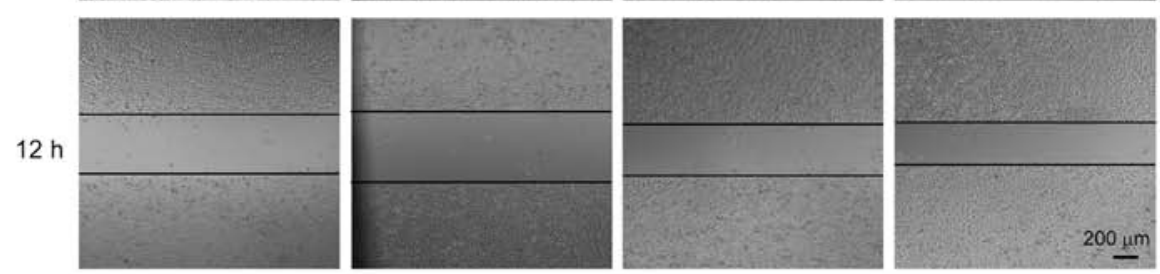

Figure 5. Effect of miR-370 on the proliferation and migration of GC cells. (A) Transwell assay indicated that miR-370 inhibited the migration of GC cells (B) Quantitative analysis of A ( $n=3$ ). (C) EdU assays demonstrated that miR-370 suppressed the proliferation of GC cells. (D) Quantitative analysis of C ( $\mathrm{n}=3$ ). (E) Evaluation of cell migration inhibition mediated by miR-370 using wound healing assay. Mimics NC refers to control mimics, inhibitors NC refers to control inhibitors; ${ }^{* *} \mathrm{p}<0.01$.

protein (Fig. 4E). Furthermore, miR-370 did not affect the mRNA levels of EGFR in the MGC-803 cells (Fig. 4F). These data demonstrated that miR-370 is a vital post-transcriptional regulator of EGFR in the GC cells, and miR-370 regulates EGFR protein expression by directly targeting the 3'-UTR of EGFR mRNA.

miR-370 suppresses cell proliferation and migration. We next assessed the biological effects of miR-370 in the GC cells in vitro. Transwell assay was performed to evaluate the migration capacity of MGC-803 cells transfected with the miR-370 mimics or inhibitors. We found that upregulation of miR-370 inhibited cell migration, whereas downregulation of miR-370 promoted migration (Fig. 5A and B). The cell proliferation activity was determined by the Cell-Light EdU Apollo DNA cell kit. As expected, a high level of miR-370 resulted in a significant suppression of cell growth capacity, while inhibition of miR-370 led to an increase in cell proliferation (Fig. 5C and D). 
We also conducted a wound healing assay to evaluate the migration ability of the transfected cells. In accordance with the results of the Transwell assay, overexpression of miR-370 showed a lower rate of migration and knockdown of miR-370 caused an increase in cell migration (Fig. 5E). These results demonstrated that miR-370 is an oncosuppressor-miRNA and plays an important role in the biological process in GC cells.

\section{Discussion}

In the present study, we obtained a number of results regarding EGFR and miR-370 in gastric cancer (GC). EGFR protein expression was controlled by post-transcriptional regulation. An miRNA regulatory mechanism was involved in this process. The biological behavior of GC cells was manipulated by altering the levels of miR-370. Our results supported these suggestions. The expression level of EGFR protein in GC tissues was not consistent with that of the mRNA level in comparison to paired adjacent non-cancerous tissues. Bioinformatics tools and dual-luciferase assay validated that miR-370 is a regulator of EGFR. Overexpression of miR-370 suppressed the proliferation and migration of the GC cells. In addition, downregulation of miR-370 promoted the proliferation and migration of the GC cells.

The role of EGFR in the pathogenesis of human malignancy has been established in a variety of cancers (26). Previous studies have shown that EGFR signaling pathways are frequently dysregulated in GC and anti-EGFR is a potential therapeutic target $(6,7)$. The present study also showed that the suppression of EGFR can inhibit the proliferation and migration of GC cells. Although, to date, all the randomized controlled phase III trials have failed to demonstrate any efficacy of anti-EGFR therapy, subpopulation analysis of the recruited patients showed that patients with EGFR gene copy number gain or protein overexpression may benefit from anti-EGFR therapy in GC (8-10). Therefore, EGFR is still a strong candidate for molecular-targeted therapy. A better understanding of the molecular mechanism that regulates EGFR protein expresion, may provide new biomarkers for predicting the clinical benefits of anti-EGFR therapy.

The expression of EGFR protein in GC is regulated at a post-transcription level. Previous studies have shown that the level of EGFR protein expression in GC is inconsistent with that of the gene. EGFR protein overexpression was detected in $\sim 30 \%$ of GC samples and one of the main causes was EGFR gene amplification (12). Kim et al found that $27.4 \%$ of GC tissues from 511 patients showed EGFR protein overexpression by IHC. However, only $2.3 \%$ of cases had gene amplification by FISH (12). Similar result from Higaki et al showed that EGFR gene amplification was not completely identical to that of IHC (2+-3+) and EGFR gene copy number gain was a more accurate prognostic bimomarker than EGFR protein overexpression in patients with GC (11). This phenomenon of inconsistency suggests that post-transcriptional regulation may exist in the expression of EGFR, although promoter methylation has been proved to be one of the mechanisms for the high expression of EGFR protein in GC (27).

As miRNA-mediated regression of mRNA transcripts is thought to be one of the most important modes of post-transcriptional regulation, we speculated that specific miRNAs may be involved in the process of EGFR protein expression in $\mathrm{GC}$ and we found that miR-370 acted as a negative regulator of EGFR in the present study. Actually, various previous studies have confirmed that the expression of EGFR protein is regulated by several miRNAs in lung (28), breast (29) and also in colorectal cancer (30). In addition, miR-370 was reported to function as a tumor suppressor in colorectal cancer by suppressing EGFR signaling (20). However, to the best of our knowledge, this is the first attempt to investigate the miR-370-EGFR pathway in GC tissues and cells.

Expression of miR-370 has been found to be dysregulated in several types of cancers. Low expression of miR-370 was found in non-small cell lung cancer and upregulation of miR-370 inhibited cell proliferation and induced cell apoptosis (31). Wu et al reported that miR-370 was aberrantly expressed in laryngeal squamous cell carcinoma (LSCC) and miR-370 may function as a tumor suppressor in LSCC through downregulation of FoxM1 (32). In addition, downregulation of miR-370 has also been noted in other malignancies such as cholangiocellular carcinoma (33), ovarian cancer (34) and oral carcinoma (35). Our research found that miR-370 was also downregulated in GC and could function as a tumor suppressor via targeting EGFR. This is consistent with the result from one previous study in which upregulation of miR-370 promoted cell apoptosis and inhibited proliferation via targeting PTEN in human GC (21). However, studies also found that upregulation of miR-370 contributed to the progression of gastric carcinoma $(36,37)$. The explanation for this may be due to the different functions of the targeted gene. Furthermore, one gene could be modulated by several miRNAs. For instance, miR-7 (38) and miR-34a (39) were both verified to be suppressors of EGFR in GC. Therefore, regulatory mechanisms of the targeted genes and the relevant miRNAs in GC need further investigation.

In conclusion, we demonstrated that EGFR protein expression is regulated by miR-370 and the miR-370-EGFR pathway is involved in the process of cell growth and migration in GC. The present study may provide new information concerning the molecular mechanism that regulates EGFR protein expression in GC and may be of important clinical significance to guide targeted therapy.

\section{Acknowledgements}

The present study was funded by grants from the National Natural Science Foundation of China (nos. 81372394, 81602158, 81572321 and 81602156), and Tianjin Health and Family Planning Commission Foundation of Science and Technology (15KG142). This work was also funded by Tianjin Science Foundation (no. 15JCYBJC28200) and Doctoral Foundation of Tianjin Medical University Cancer Institute and Hospital (B1502).

\section{References}

1. Allemani C, Weir HK, Carreira H, Harewood R, Spika D, Wang XS, Bannon F, Ahn JV, Johnson CJ, Bonaventure A, et al; CONCORD Working Group: Global surveillance of cancer survival 1995-2009: Analysis of individual data for 25,676,887 patients from 279 population-based registries in 67 countries (CONCORD-2). Lancet 385: 977-1010, 2015. 
2. Dixon M, Mahar AL, Helyer LK, Vasilevska-Ristovska J, Law C and Coburn NG: Prognostic factors in metastatic gastric cancer: Results of a population-based, retrospective cohort study in Ontario. Gastric Cancer 19: 150-159, 2016.

3. Strong VE, Wu AW, Selby LV, Gonen M, Hsu M, Song KY, Park CH, Coit DG, Ji JF and Brennan MF: Differences in gastric cancer survival between the U.S. and China. J Surg Oncol 112: $31-37,2015$

4. $\mathrm{Xu} \mathrm{W}$, Yang $\mathrm{Z}$ and $\mathrm{Lu} \mathrm{N}$ : Molecular targeted therapy for the treatment of gastric cancer. J Exp Clin Cancer Res 35: 1, 2016.

5. Yarden Y and Pines G: The ERBB network: At last, cancer therapy meets systems biology. Nat Rev Cancer 12: 553-563, 2012.

6. Terashima M, Kitada K, Ochiai A, Ichikawa W, Kurahashi I, Sakuramoto S, Katai H, Sano T, Imamura $\mathrm{H}$ and Sasako M; ACTS-GC Group: Impact of expression of human epidermal growth factor receptors EGFR and ERBB2 on survival in stage II/III gastric cancer. Clin Cancer Res 18: 5992-6000, 2012.

7. Yang W, Raufi A and Klempner SJ: Targeted therapy for gastric cancer: Molecular pathways and ongoing investigations. Biochim Biophys Acta 1846: 232-237, 2014.

8. Fontana $\mathrm{E}$ and Smyth EC: Novel targets in the treatment of advanced gastric cancer: A perspective review. Ther Adv Med Oncol 8: 113-125, 2016.

9. Jiang Z, Li C, Li F and Wang X: EGFR gene copy number as a prognostic marker in colorectal cancer patients treated with cetuximab or panitumumab: A systematic review and meta analysis. PLoS One 8: e56205, 2013.

10. Pirker R, Pereira JR, von Pawel J, Krzakowski M, Ramlau R, Park K, de Marinis F, Eberhardt WE, Paz-Ares L, Störkel S, et al: EGFR expression as a predictor of survival for first-line chemotherapy plus cetuximab in patients with advanced non-small-cell lung cancer: Analysis of data from the phase 3 FLEX study. Lancet Oncol 13: 33-42, 2012.

11. Higaki E, Kuwata T, Nagatsuma AK, Nishida Y, Kinoshita T, Aizawa M, Nitta H, Nagino M and Ochiai A: Gene copy number gain of EGFR is a poor prognostic biomarker in gastric cancer: Evaluation of 855 patients with bright-field dual in situ hybridization (DISH) method. Gastric Cancer 19: 63-73, 2016.

12. Kim MA, Lee HS, Lee HE, Jeon YK, Yang HK and Kim WH: EGFR in gastric carcinomas: Prognostic significance of protein overexpression and high gene copy number. Histopathology 52 : 738-746, 2008

13. Bartel DP: MicroRNAs: Genomics, biogenesis, mechanism, and function. Cell 116: 281-297, 2004.

14. Filipowicz W, Bhattacharyya SN and Sonenberg N: Mechanisms of post-transcriptional regulation by microRNAs: Are the answers in sight? Nat Rev Genet 9: 102-114, 2008.

15. Friedman JM and Jones PA: MicroRNAs: Critical mediators of differentiation, development and disease. Swiss Med Wkly 139: 466-472, 2009

16. Calin GA, Sevignani C, Dumitru CD, Hyslop T, Noch E, Yendamuri S, Shimizu M, Rattan S, Bullrich F, Negrini M, et al: Human microRNA genes are frequently located at fragile sites and genomic regions involved in cancers. Proc Natl Acad Sci USA 101: 2999-3004, 2004.

17. Li X, Zhang Z, Yu M, Li L, Du G, Xiao W and Yang H: Involvement of miR-20a in promoting gastric cancer progression by targeting early growth response 2 (EGR2). Int J Mol Sci 14: 16226-16239, 2013.

18. Li H, Xie S, Liu X, Wu H, Lin X, Gu J, Wang H and Duan Y: Matrine alters microRNA expression profiles in SGC-7901 human gastric cancer cells. Oncol Rep 32: 2118-2126, 2014.

19. Riquelme I, Tapia O, Leal P, Sandoval A, Varga MG, Letelier P, Buchegger K, Bizama C, Espinoza JA, Peek RM, et al: miR-101-2, miR-125b-2 and miR-451a act as potential tumor suppressors in gastric cancer through regulation of the PI3K/AKT/mTOR pathway. Cell Oncol 39: 23-33, 2016.

20. El-Daly SM, Abba ML, Patil N and Allgayer H: miRs-134 and -370 function as tumor suppressors in colorectal cancer by independently suppressing EGFR and PI3K signaling. Sci Rep 6: 24720,2016
21. Zeng Y, Fu M, Wu GW, Zhang AZ, Chen JP, Lin HY, Fu YA, Jia J, Cai ZD, Wu XJ, et al: Upregulation of microRNA-370 promotes cell apoptosis and inhibits proliferation by targeting PTEN in human gastric cancer. Int J Oncol 49: 1589-1599, 2016.

22. John B, Enright AJ, Aravin A, Tuschl T, Sander C and Marks DS: Human MicroRNA targets. PLoS Biol 2: e363, 2004.

23. Lewis BP, Burge CB and Bartel DP: Conserved seed pairing, often flanked by adenosines, indicates that thousands of human genes are microRNA targets. Cell 120: 15-20, 2005.

24. Krek A, Grün D, Poy MN, Wolf R, Rosenberg L, Epstein EJ, MacMenamin P, da Piedade I, Gunsalus KC, Stoffel M, et al: Combinatorial microRNA target predictions. Nat Genet 37: 495-500, 2005

25. Yang S, Luo A, Hao X, Lai Z, Ding T, Ma X, Mayinuer M, Shen W, Wang X, Lu Y, et al: Peroxiredoxin 2 inhibits granulosa cell apoptosis during follicle atresia through the NFKB pathway in mice. Biol Reprod 84: 1182-1189, 2011.

26. Ciardiello $\mathrm{F}$ and Tortora G: EGFR antagonists in cancer treatment. N Engl J Med 358: 1160-1174, 2008

27. Weng X, Zhang H, Ye J, Kan M, Liu F, Wang T, Deng J, Tan Y, He L and Liu Y: Hypermethylated Epidermal growth factor receptor (EGFR) promoter is associated with gastric cancer. Sci Rep 5: 10154, 2015.

28. Weiss GJ, Bemis LT, Nakajima E, Sugita M, Birks DK, Robinson WA, Varella-Garcia M, Bunn PA Jr, Haney J, Helfrich BA, et al: EGFR regulation by microRNA in lung cancer: Correlation with clinical response and survival to gefitinib and EGFR expression in cell lines. Ann Oncol 19: 1053-1059, 2008.

29. Uhlmann S, Mannsperger H, Zhang JD, Horvat EA, Schmidt C, Küblbeck M, Henjes F, Ward A, Tschulena U, Zweig K, et al: Global microRNA level regulation of EGFR-driven cell-cycle protein network in breast cancer. Mol Syst Biol 8: 570, 2012.

30. Mlcochova J, Faltejskova P, Nemecek R, Svoboda M and Slaby O: MicroRNAs targeting EGFR signalling pathway in colorectal cancer. J Cancer Res Clin Oncol 139: 1615-1624, 2013.

31. Chen T, Gao F, Feng S, Yang T and Chen M: MicroRNA-370 inhibits the progression of non-small cell lung cancer by downregulating oncogene TRAF4. Oncol Rep 34: 461-468, 2015.

32. Yungang W, Xiaoyu L, Pang T, Wenming L and Pan X: miR-370 targeted FoxM1 functions as a tumor suppressor in laryngeal squamous cell carcinoma (LSCC). Biomed Pharmacother 68: 149-154, 2014.

33. Meng F, Wehbe-Janek H, Henson R, Smith $\mathrm{H}$ and Patel T: Epigenetic regulation of microRNA-370 by interleukin- 6 in malignant human cholangiocytes. Oncogene 27: 378-386, 2008.

34. Chen XP, Chen YG, Lan JY and Shen ZJ: MicroRNA-370 suppresses proliferation and promotes endometrioid ovarian cancer chemosensitivity to cDDP by negatively regulating ENG. Cancer Lett 353: 201-210, 2014

35. Chang KW, Chu TH, Gong NR, Chiang WF, Yang CC, Liu CJ, Wu $\mathrm{CH}$ and Lin SC: $m i R-370$ modulates insulin receptor substrate-1 expression and inhibits the tumor phenotypes of oral carcinoma. Oral Dis 19: 611-619, 2013.

36. Lo SS, Hung PS, Chen JH, Tu HF, Fang WL, Chen CY, Chen WT, Gong NR and Wu CW: Overexpression of $m i R-370$ and downregulation of its novel target TGF $\beta$-RII contribute to the progression of gastric carcinoma. Oncogene 31: 226-237, 2012.

37. Fan C, Liu S, Zhao Y, Han Y, Yang L, Tao G, Li Q and Zhang L: Upregulation of miR-370 contributes to the progression of gastric carcinoma via suppression of FOXO1. Biomed Pharmacother 67: 521-526, 2013.

38. Xie J, Chen M, Zhou J, Mo MS, Zhu LH, Liu YP, Gui QJ, Zhang L and Li GQ: miR-7 inhibits the invasion and metastasis of gastric cancer cells by suppressing epidermal growth factor receptor expression. Oncol Rep 31: 1715-1722, 2014.

39. Liu G, Jiang C, Li D, Wang R and Wang W: MiRNA-34a inhibits EGFR-signaling-dependent MMP7 activation in gastric cancer. Tumour Biol 35: 9801-9806, 2014. 